\title{
Direct Measurement of the Cosmic-Ray Iron Spectrum with the Dark Matter Particle Explorer
}

Zhi-hui Xu, ${ }^{a, b, *}$ Francesca Alemanno,${ }^{c, d}$ Peng-xiong Ma, ${ }^{a}$ Leandro Silveri ${ }^{c, d}$ and Qiang Yuan ${ }^{a, b}$ on behalf of the DAMPE Collaboration

(a complete list of authors can be found at the end of the proceedings)

${ }^{a}$ Key Laboratory of Dark Matter and Space Astronomy, Purple Mountain Observatory, Chinese Academy of Sciences, No.10 Yuanhua Road, Qixia District, Nanjing 210034, China

${ }^{b}$ School of Astronomy and Space Science, University of Science and Technology of China,Hefei 230026, China

${ }^{c}$ Gran Sasso Science Institute (GSSI), Via Iacobucci 2, I-67100 L'Aquila, Italy

${ }^{d}$ Istituto Nazionale di Fisica Nucleare (INFN) -Laboratori Nazionali del Gran Sasso, I-67100 Assergi, L'Aquila, Italy

E-mail: xuzh@pmo.ac.cn

Dark Matter Particle Explorer(DAMPE) is a calorimetric-type, satellite-borne detector for observations of high energy electrons, gamma-rays, and cosmic-ray nuclei. Using five years data collected with DAMPE from January 1, 2016 to December 31, 2020, we analyzed the spectrum of iron. Detailed studies of the fragmentation of iron in the detector have been performed using Monte Carlo simulations.

$37^{\text {th }}$ International Cosmic Ray Conference (ICRC 2021)

July 12 th - 23rd, 2021

Online - Berlin, Germany

\footnotetext{
${ }^{*}$ Presenter
} 


\section{Introduction}

The precise measurements of primary cosmic rays provide important information on the origin, acceleration, and propagation processes of cosmic rays in the galaxy. Among the abundant cosmic ray nuclei, iron has the larger interaction cross sections and relative smaller Larmor radius.So iron spectrum is very important in shedding light on source of cosmic rays, propagation and interaction with interstellar medium[1-4].

Up to now, there have been many measurements of the iron flux, such as balloon-borne experiment ATIC-2[5], and space-borne experiments HEAO-3[6], TRACER06[7] , and magnetic spectrometric experiment AMS-2[8].

DAMPE is a calorimetric-type instrument, which has a large acceptance and good charge resolution. DAMPE has continuously collected more than 10 billions events since launched on Dec. 17, 2015. In this report we present the analysis procedure of iron flux. We also do detailed studies of the fragmentation of iron in the detector using Monte Carlo simulations.

\section{DAMPE instrument}

The DAMPE consists of 4 sub-detectors. The Plastic Scintillator Detector (PSD) is used to measure particle charge, it can also be used as an anti-coincidence detector for $\gamma$-rays. The Silicon Tungsten tracKer-converter (STK) is used for trajectory measurement as well as charge measurement. The $\mathrm{Bi}_{3} \mathrm{Ge}_{4} \mathrm{O}_{12}(\mathrm{BGO})$ electromagnetic calorimeter plays a key role in energy measurement as well as electron-hadron identification. The NeUtron Detector (NUD) provide further electron-hadron separation[9]. Four sub-detectors have been operating stably and in good working conditions[10, $11]$.

\section{Fragmentation and quenching of iron in detector}

The inelastic scattering cross section of nucleus is proportional to the $2 / 3$ power of the mass number of nucleus. The binding energy per nucleon of iron is $8.6 \mathrm{MeV}$. The energy required for iron nuclei fragmentation is $0.45 \mathrm{GeV}$. When Fe nuclei pass through the material on the top of the detector, they are easily broken. So the inelastic scattering is important for iron spectrum analysis. Most of irons fragment into light nuclei, such as protons and helium nuclei. It is found that the fragmentation channels of iron in different energy ranges are obviously different in FTFP_BERT model. Although the total inelastic scattering cross section varies little with energy, the fragmentation channels are quite different. Iron with tend to fragment into heavier secondary particles around $100 \mathrm{GeV}$ in FTFP model.

Fig. [1] (left) shows the total fragmentation probability in PSD detector by using FTFP and FLUKA models. Fig. [1] (right) shows the ratio of Fe fragmented into sub-Fe( $\mathrm{Sc}, \mathrm{Ti}, \mathrm{V}, \mathrm{Cr}, \mathrm{Mn}$ ) channels to the total fragmentation probability in PSD detector by using FTFP and FLUKA models. The probability of iron fragmentation in the FTFP model is higher. So the track selection efficiency in FTFP model is relatively lower, but near $100 \mathrm{GeV}$, the iron has a higher probability of fragmentation into a sub-iron nuclei, for which we can also reconstruct the track.

Fig. [2] shows the distribution of Stop_Z (Z-direction coordinates of first inelastic scattering) with energy.The fragmentation probability of FTFP bigger then FLUKA, so the in FLUKA modle, 

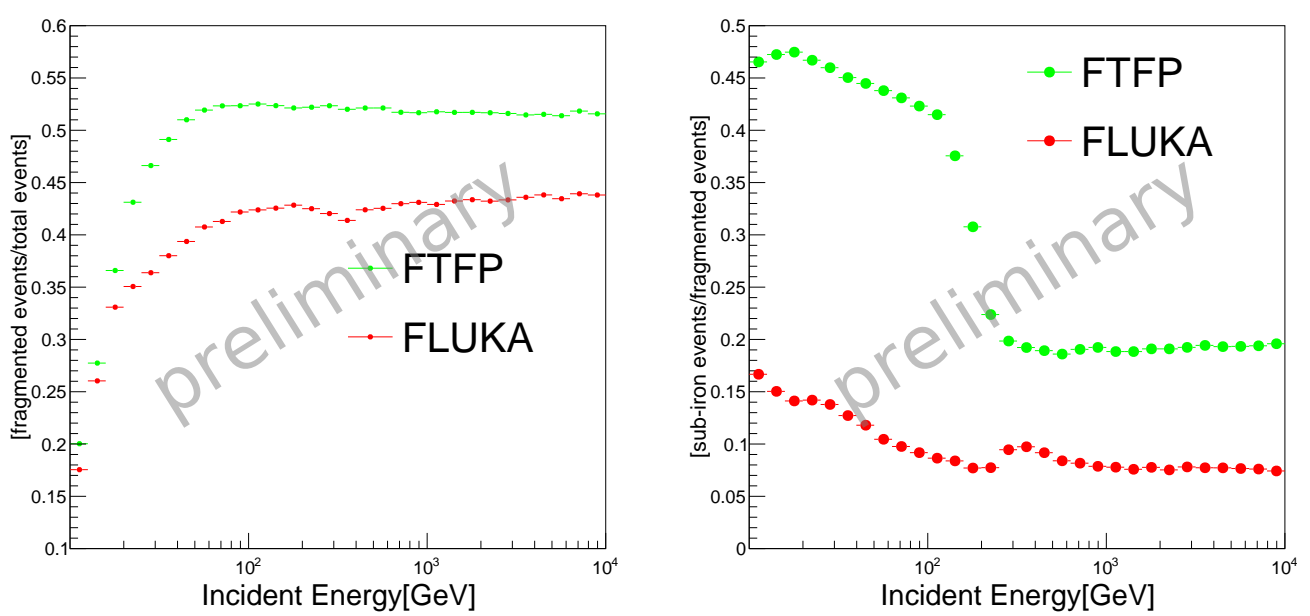

Figure 1: Left-the probability fragment of iron in PSD. Right-the ratio of fragmented into sub-iron to total fragmentation result.
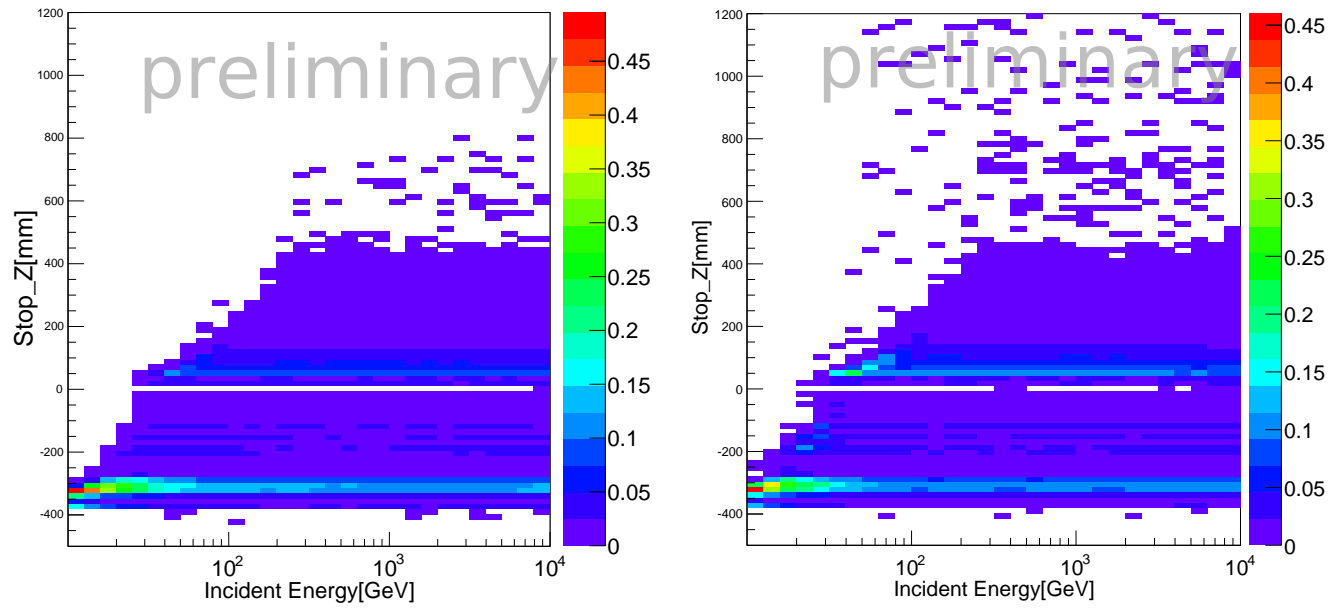

Figure 2: the position of first inelastic scattering of iron in detector. Left-FTFP model. Right-FLUKA model.

more events fragmented after path through BGO detector. When irons passes through the 4-layer PSD, it loses approximately $5.4 \mathrm{GeV}$ of energy. Therefore, the iron with dozens of $\mathrm{GeV}$ incident energy loss a large portion of energy.Consequently most of the low-energy irons disappear in the top of the detector. If iron can not pass through the STK, the track will not be reconstructed, and if they can not pass through the first four layers of BGO, the trigger condition may not be satisfied. Therefore, the selection efficiency drop quickly with the decrease of the incident energy.

Fig. [3] show the energy deposition fraction $\left(E_{D e p} / E_{\text {inc }}\right)$ from 80 to $160 \mathrm{GeV}$ for FTFP model. In this energy range, irons have more probability fragmented into sub-rions, which loses relatively little energy. When irons fragmented in PSD or STK into sub-irons, the secondary particles still could reconstruct STK tracks and carry much energy into BGO detector. If irons fragmented in 

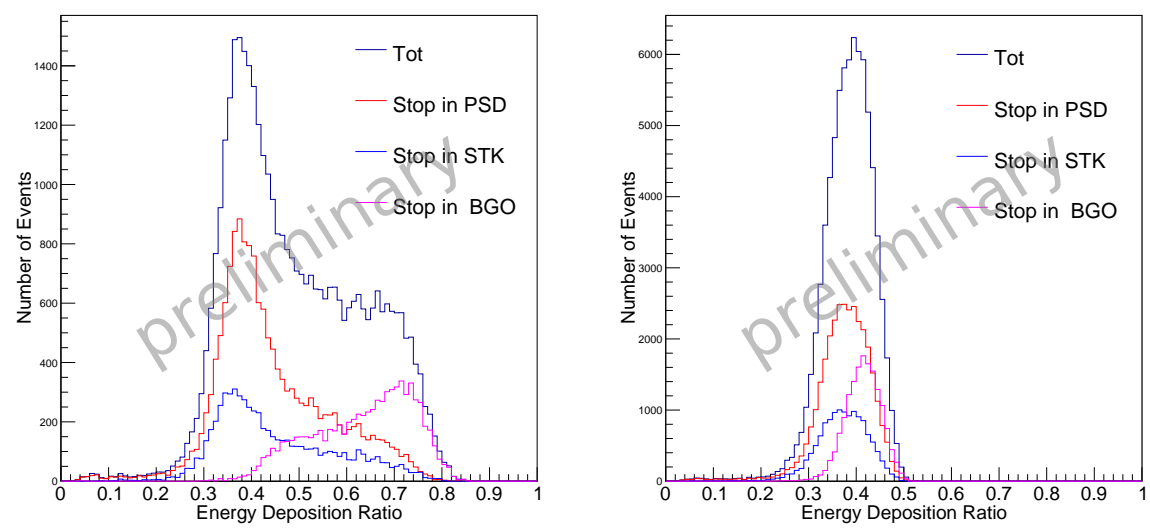

Figure 3: Energy Deposited ratio of iron MC sample with energy from 80 to $160 \mathrm{GeV}$. left-Model of FTFP, right-Model of FTFP with quenching.
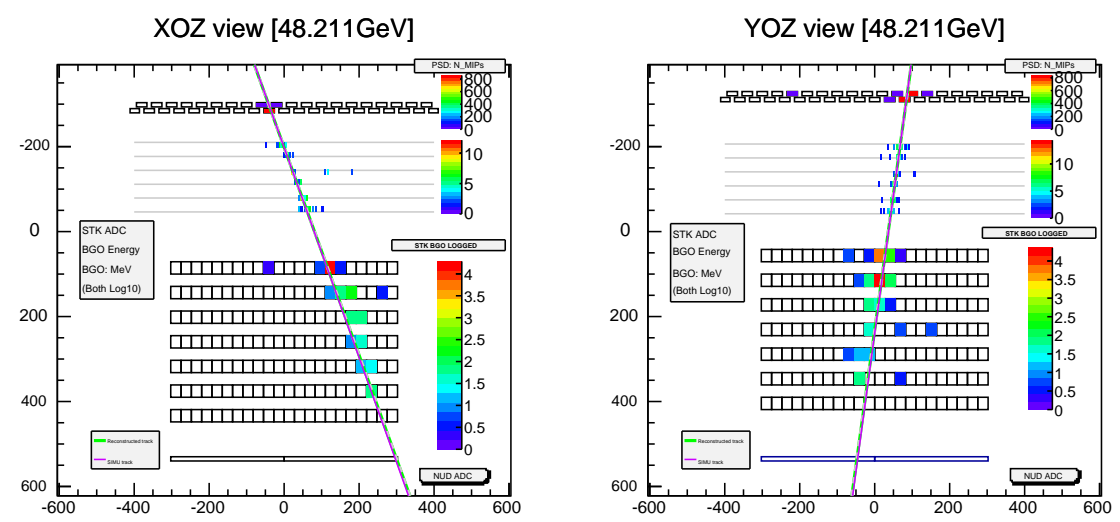

Figure 4: Topological graph of iron fragmented in BGO detector, which deposit relative much energy in BGO detector.left-view of $X O Z$ direction, right-view of $Y O Z$ direction,

PSD or STK into Heliums or Protons, it is very difficult to reconstruct STK tracks, and secondary particles carry fewer energy into BGO detector. The position of fragmentation also affect the track reconstruction and energy deposition ratio. In this energy range, the particles fragmented in BGO detector can deposit the most energy in BGO as shown in Fig. [4], and can easily reconstruct STK track. The particles fragmented in STK detector can deposit the fewer energy in BGO as shown in Fig. [5], and is difficult to reconstruct STK track.

The final result of the energy deposition ratio is shown in Fig. [6](right). Not only is the difference between different models very large, but the difference in the quenching effect caused by the fragmentation channel is also very large.

\section{Data analysis}

We use 5 years of data from January 1, 2016 to December 31, 2021. The datasets collected in the South Atlantic Anomaly(SAA) region are excluded. The live time of this analysis is about 

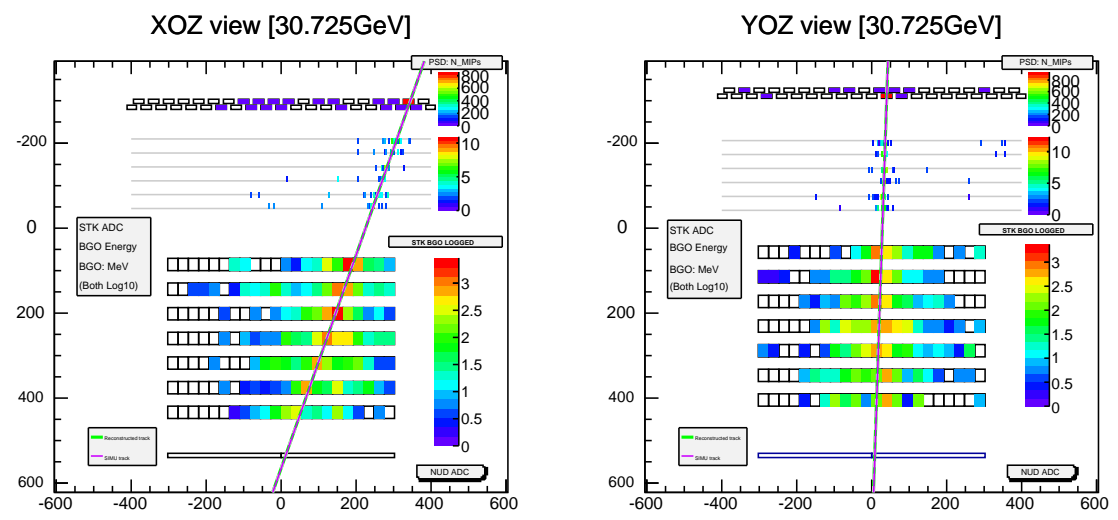

Figure 5: Topological graph of iron fragmented in STK detector, which deposit relative fewer energy in BGO detector.left-view of $X O Z$ direction, right-view of $Y O Z$ direction,

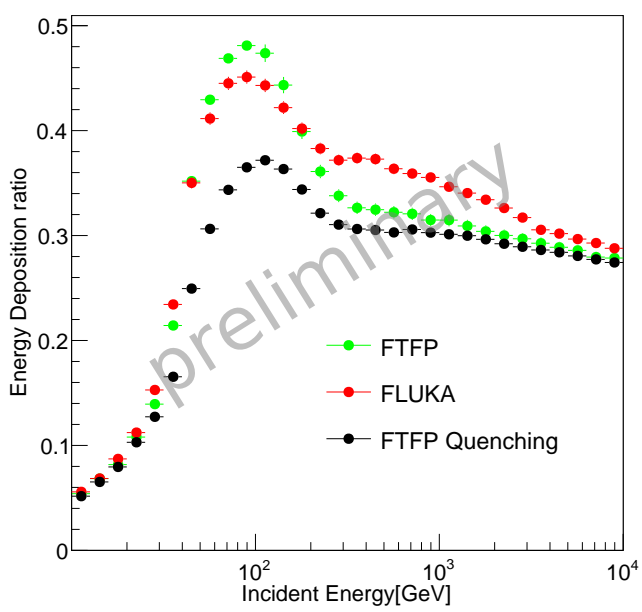

Figure 6: The Energy deposition ratio, which affected by quenching effect.

$1.2 \times 10^{8}$ seconds. Monte-Carlo (MC) simulation datasets were generated with Geant4 toolkit with the physics model FTFP_BERT, quenching effect on the BGO calorimeter is also taken into account.In order to reconstruct the spectrum of iron, we used MC data as template to estimate the contamination, then we reconstruct the original spectrum by unfolding the measured spectrum.

\subsection{Event Selection}

- Pre-selection.

We select events with deposited energy in BGO calorimeter larger than $80 \mathrm{GeV}$, because low energy events deposit too much energy in PSD and STK. Therefore energy resolution must significantly decrease when energy deposition is lower than $80 \mathrm{GeV}$ in BGO. The good events should satisfy several requirements. First of all, the events should meet the High Energy Trigger (HET) requirement, and at lest there is one STK track. 


\section{- STK Track selection.}

STK detector consists of 12 layers silicon strip detectors, when particle passes through the silicon, the detector records clusters in hit points, then we can use these clusters to construct track with Kalman filter algorithm. In most probable situations, there exist more than one tracks constructed for one incident primary cosmic ray particle, but only one is true. We use several criteria to select the best track. Firstly the track at least has 8 clusters to ensure that the track is reliable. Secondly, we require the STK track passes through the PSD bar which has the maximum read-out in the whole layers. It is because the back of splash particles can not deposit comparable energy with iron itself in PSD detector. Furthermore, we still require that the reduced $c h i^{2}$ of track is less than 50 to exclude those of bad quality. Finally, we select the track which has the biggest average ADC read-out in STK detector.

\section{- Charge selection.}

PSD consists of two layers placed in a cross staggered configuration (Y-view for layer-1 and $\mathrm{X}$-view for layer-2), with 41 plastic scintillator logs in each layer. The energy deposition of charged particles is proportional to $Z^{2}$ if the path length is given. The energy deposition is about $2 \mathrm{MeV}$ for proton minimum ionization particles (MIPs) cross one layer PSD vertically(the path length is $1 \mathrm{~cm}$ ). The particles are required to pass through both X-layer and Y-layer. Meanwhile, it is required that the length in each PSD bar is larger than $5 \mathrm{~mm}$, and if the charges difference derived from the two layers differ by larger $10 \%$, we will take the larger one as the charge value[12,13]. The charge readout has a series of calibrations, such as hit position calibration, fluorescence attenuation correction and alignment correction, as well as the calibration of different layers of readout difference. We fixed the peak value of the iron at 26, and fixed the peak value of the charge readout of sub-iron at the corresponding nominal value. As a result, after a series of calibrations, the peak values of all elements does not change with energy. The iron flux is the highest among cosmic rays with $Z>20$, and pollution mainly comes from $\operatorname{Mn}(Z=25)$, so we select candidate iron with charge from 25.5 to 27.2. Finally, we exclude the events for which the charges of both layers are less than 10. This can directly eliminate the case of recoil to STK and PSD after being broken at the bottom of the detector.

\subsection{Particle identification and background}

In order to effectively distinguish between iron and sub-irons, and estimate the pollution by super-iron elements, we used 10 Landau convolution Gaussian functions to fit flight data from $\mathrm{Z}=19(\mathrm{~K})$ to $\mathrm{Z}=28$ ( Ni) elements, and then use the simulated data as a template to fit the flight data. Fig. [7] shows the results of template fitting from 158 to $200 \mathrm{GeV}$. With template fitting, the contaminations of other elements are calculated and shown in right of Fig. [7].

\section{Summary and outlook}

Detailed studies of the fragmentation of iron in the detector have been performed using Monte Carlo simulations. It is found that the fragmentation and quenching affect events reconstruction and the efficiency of data selection. DAMPE has very accurate particle identification capability for 

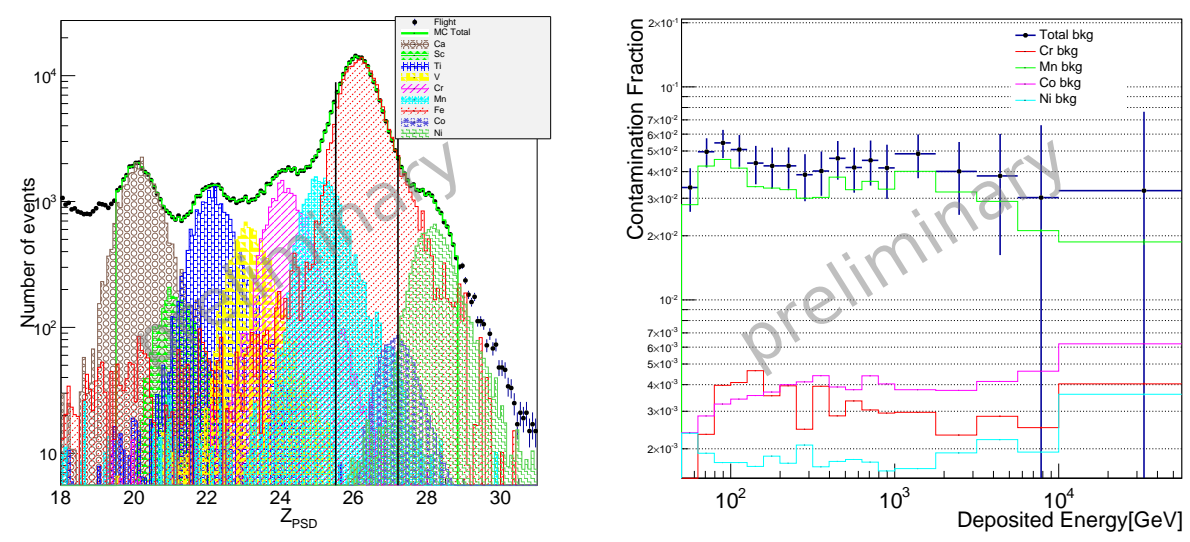

Figure 7: left-template fiting of data.Black point are the flight data, green solid line are simulation data.rightcontamination.Black point represent the total contamination, and the green circle is the contamination from $\mathrm{Mn}$

Fe and Sub-Fe elements. But there are still a lot of detailed work to be done. In the future, we will give an iron spectrum up to few $\mathrm{TeV} / n$ and improve the precision at higher energies.

\section{Acknowledgement}

The DAMPE mission was funded by the strategic priority science and technology projects in space science of Chinese Academy of Sciences. In China the data analysis is supported by the National Key Research and Development Program of China (No. 2016YFA0400200), the National Natural Science Foundation of China (Nos. 11921003, 11622327, 11722328, 11851305, U1738205, U1738206, U1738207, U1738208, U1738127), the strategic priority science and technology projects of Chinese Academy of Sciences (No. XDA15051100), the 100 Talents Program of Chinese Academy of Sciences, the Young Elite Scientists Sponsorship Program by CAST (No. YESS20160196), and the Program for Innovative Talents and Entrepreneur in Jiangsu. In Europe the activities and data analysis are supported by the Swiss National Science Foundation (SNSF), Switzerland, the National Institute for Nuclear Physics (INFN), Italy, and the European Research Council (ERC) under the European Union's Horizon 2020 research and innovation programme (No. 851103).

\section{References}

[1] Castellina, A. \& Donato, F. 2005, Astroparticle Physics, 24, 146. doi:10.1016/j.astropartphys.2005.06.006

[2] Strong, A. W., Moskalenko, I. V., \& Ptuskin, V. S. 2007, Annual Review of Nuclear and Particle Science, 57, 285. doi:10.1146/annurev.nucl.57.090506.123011

[3] Blasi, P. 2013, , 21, 70. doi:10.1007/s00159-013-0070-7 
[4] Grenier, I. A., Black, J. H., \& Strong, A. W. 2015, , 53, 199. doi:10.1146/annurev-astro082214-122457

[5] Panov, A. D., Adams, J. H., Ahn, H. S., et al. 2009, Bulletin of the Russian Academy of Sciences, Physics, 73, 564. doi:10.3103/S1062873809050098

[6] Engelmann, J. J., Ferrando, P., Soutoul, A., et al. 1990, , 233, 96

[7] Obermeier, A., Ave, M., Boyle, P., et al. 2011, , 742, 14. doi:10.1088/0004-637X/742/1/14

[8] Aguilar, M., Cavasonza, L. A., Allen, M. S., et al. 2021, , 126, 041104. doi:10.1103/PhysRevLett.126.041104

[9] Chang, J., Ambrosi, G., An, Q., et al. 2017, Astroparticle Physics, 95, 6. doi:10.1016/j.astropartphys.2017.08.005

[10] Tykhonov, A., Ambrosi, G., Asfandiyarov, R., et al. 2019, Nuclear Instruments and Methods in Physics Research A, 924, 309. doi:10.1016/j.nima.2018.06.036

[11] Ambrosi, G., An, Q., Asfandiyarov, R., et al. 2019, Astroparticle Physics, 106, 18. doi:10.1016/j.astropartphys.2018.10.006

[12] Ding, M., Zhang, Y.-P., Zhang, Y.-J., et al. 2019, Research in Astronomy and Astrophysics, 19, 047. doi:10.1088/1674-4527/19/3/47

[13] Dong, T., Zhang, Y., Ma, P., et al. 2019, Astroparticle Physics, 105, 31. doi:10.1016/j.astropartphys.2018.10.001

[14] D’Agostini, G. 1995, Nuclear Instruments and Methods in Physics Research A, 362, 487. doi:10.1016/0168-9002(95)00274-X 


\section{Full Authors List: DAMPE Collaboration}

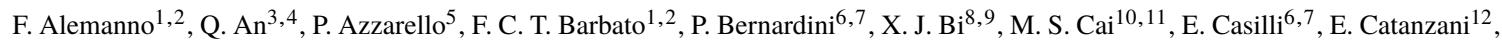
J. Chang ${ }^{10,11}$, D. Y. Chen ${ }^{9,10}$, J. L. Chen ${ }^{13}$, Z. F. Chen ${ }^{10,11}$, M. Y. Cui ${ }^{10}$, T. S. Cui ${ }^{14}$, Y. X. Cui ${ }^{10,11}$, H. T. Dai ${ }^{3,4}$, A. De Benedittis ${ }^{6,7}$,

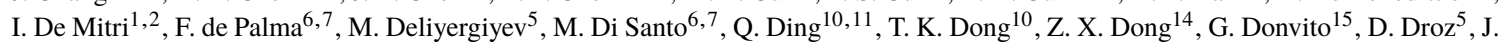
L. Duan ${ }^{13}$, K. K. Duan ${ }^{10}$, D. D’Urso ${ }^{12,1}$, R. R. Fan ${ }^{8}$, Y. Z. Fan ${ }^{10,11}$, K. Fang ${ }^{8}$, F. Fang ${ }^{13}$, C. Q. Feng ${ }^{3,4}$, L. Feng ${ }^{10}$, P. Fusco ${ }^{15,16}$, M.

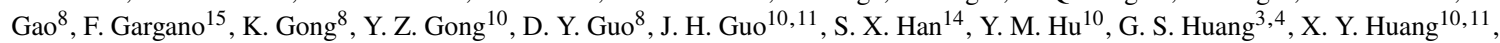
Y. Y. Huang ${ }^{10}$, M. Ionica ${ }^{12}$, W. Jiang ${ }^{10,11}$, J. Kong ${ }^{13}$, A. Kotenko ${ }^{5}$, D. Kyratzis ${ }^{1,2}$, S. J. Lei ${ }^{10}$, W. H. Li ${ }^{10,11}$, W. L. Li ${ }^{14}$, X. Li $^{10}$,

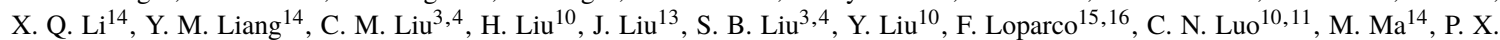

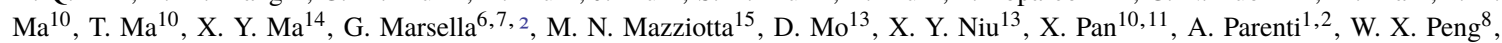
X. Y. Peng ${ }^{10}$, C. Perrina ${ }^{5,3}$, R. Qiao $^{8}$, J. N. Rao ${ }^{14}$, A. Ruina ${ }^{5}$, M. M. Salinas ${ }^{5}$, G. Z. Shang ${ }^{14}$, W. H. Shen ${ }^{14}$, Z. Q. Shen ${ }^{10}$, Z. T. $\mathrm{Shen}^{3,4}$, L. Silveri ${ }^{1,2}$, J. X. Song ${ }^{14}$, M. Stolpovskiy ${ }^{5}$, H. Su ${ }^{13}$, M. Su${ }^{17}$, H. R. Sun ${ }^{3,4}$, Z. Y. Sun ${ }^{13}$, A. Surdo ${ }^{7}$, X. J. Teng ${ }^{14}$, A. $^{2}$

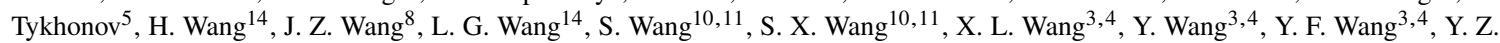
Wang ${ }^{10}$, D. M. Wei ${ }^{10,11}$, J. J. Wei ${ }^{10}$, Y. F. Wei ${ }^{3,4}$, D. Wu ${ }^{8}$, J. Wu ${ }^{10,11}$, L. B. Wu ${ }^{1,2}$, S. S. Wu ${ }^{14}, \mathrm{X} \mathrm{Wu}^{5}$, Z. Q. Xia ${ }^{10}$, E. H. Xu ${ }^{3,4}$, H. T. $\mathrm{Xu}^{14}$, Z. H. Xu${ }^{10}, 11$, Z. L. Xu ${ }^{10}$, Z. Z. Xu ${ }^{3,4}$, G. F. Xue ${ }^{14}$, H. B. Yang ${ }^{13}$, P. Yang ${ }^{13}$, Y. Q. Yang ${ }^{13}$, H. J. Yao ${ }^{13}$, Y. H. Yu ${ }^{13}$, G. W. Yuan ${ }^{10,11}$, Q. Yuan ${ }^{10,11}$, C. Yue ${ }^{10}$, J. J. Zang ${ }^{10,4}$, F. Zhang ${ }^{8}$, S. X. Zhang ${ }^{13}$, W. Z. Zhang ${ }^{14}$, Y. Zhang ${ }^{10}$, Y. J. Zhang ${ }^{13}$, Y. L. Zhang $^{3,4}$, Y. P. Zhang ${ }^{13}$, Y. Q. Zhang ${ }^{10}$, Z. Zhang ${ }^{10}$, Z. Y. Zhang ${ }^{3,4}$, C. Zhao ${ }^{3,4}$, H. Y. Zhao ${ }^{13}$, X. F. Zhao ${ }^{14}$, C. Y. Zhou ${ }^{14}$, and Y. Zhu ${ }^{14}$

${ }^{1}$ Gran Sasso Science Institute (GSSI), Via Iacobucci 2, I-67100 L'Aquila, Italy

${ }^{2}$ Istituto Nazionale di Fisica Nucleare (INFN) -Laboratori Nazionali del Gran Sasso, I-67100 Assergi, L'Aquila, Italy

${ }^{3}$ State Key Laboratory of Particle Detection and Electronics, University of Science and Technology of China, Hefei 230026, China

${ }^{4}$ Department of Modern Physics, University of Science and Technology of China, Hefei 230026, China

${ }^{5}$ Department of Nuclear and Particle Physics, University of Geneva, CH-1211, Switzerland

${ }^{6}$ Dipartimento di Matematica e Fisica E. De Giorgi, Universita‘ del Salento, I-73100, Lecce, Italy

${ }^{7}$ Istituto Nazionale di Fisica Nucleare (INFN) - Sezione di Lecce, I-73100, Lecce, Italy

${ }^{8}$ Institute of High Energy Physics, Chinese Academy of Sciences, Yuquan Road 19B, Beijing 100049, China

${ }^{9}$ University of Chinese Academy of Sciences, Yuquan Road 19A, Beijing 100049, China

${ }^{10}$ Key Laboratory of Dark Matter and Space Astronomy, Purple Mountain Observatory, Chinese Academy of Sciences, Nanjing 210023, China

${ }^{11}$ School of Astronomy and Space Science, University of Science and Technology of China, Hefei 230026, China

${ }^{12}$ Istituto Nazionale di Fisica Nucleare (INFN) - Sezione di Perugia, I-06123 Perugia, Italy

${ }^{13}$ Institute of Modern Physics, Chinese Academy of Sciences, Nanchang Road 509, Lanzhou 730000, China

${ }^{14}$ National Space Science Center, Chinese Academy of Sciences, Nanertiao 1, Zhongguancun, Haidian district, Beijing 100190, China

${ }^{15}$ Istituto Nazionale di Fisica Nucleare (INFN) - Sezione di Bari, I-70125, Bari, Italy

${ }^{16}$ Dipartimento di Fisica “M. Merlin" dell'Universita' e del Politecnico di Bari, I-70126, Bari, Italy

${ }^{17}$ Department of Physics and Laboratory for Space Research, the University of Hong Kong, Pok Fu Lam, Hong Kong SAR, China

${ }^{1}$ Now at Universita‘ di Sassari, Dipartimento di Chimica e Farmacia, I-07100, Sassari, Italy.

${ }^{2}$ Now at Universita“ degli Studi di Palermo, Dipartimento di Fisica e Chimica "E. Segre“", via delle Scienze ed. 17, I-90128 Palermo, Italy.

${ }^{3}$ Also at Institute of Physics, Ecole Polytechnique Federale de Lausanne (EPFL), CH-1015 Lausanne, Switzerland.

${ }^{4}$ Also at School of Physics and Electronic Engineering, Linyi University, Linyi 276000, China. 